EPJ Web of Conferences 53, 08009 (2013)

DOI: $10.1051 /$ epjconf/20135308009

(C) Owned by the authors, published by EDP Sciences, 2013

\title{
Status of the program for microwave detection of cosmic rays at the Pierre Auger observatory
}

\author{
P. Facal San Luis ${ }^{1, a}$ for the Pierre Auger Collaboration ${ }^{2, b}$ \\ ${ }^{1}$ Enrico Fermi Institute, University of Chicago, Chicago IL 60637, USA \\ ${ }^{2}$ Observatorio Pierre Auger, Av. San Martín Norte 304, 5613 Malargüe, Argentina \\ (Full author list: $h$ ttp://www.auger.org/archive/authors_2012_06.html)
}

\begin{abstract}
Radiation in the microwave band from the passage of charged particles through air has been detected in the laboratory. This radiation could provide a novel technique for the detection of ultra-high energy cosmic rays over large areas, with $100 \%$ duty cycle and virtually no atmospheric attenuation. Detection of extensive air showers in the $\mathrm{GHz}$ band is being actively pursued at the Pierre Auger Observatory. The status and first results of the R\&D activities on microwave detection at the Pierre Auger Observatory are presented.
\end{abstract}

\section{INTRODUCTION}

The Pierre Auger Observatory [1] is dedicated to the study of Ultra High Energy Cosmic Rays (UHECRs) with a $3000 \mathrm{~km}^{2}$ hybrid detector installed in Malargüe, Argentina. The Auger Collaboration is pursuing several activities with the objective of enhancing the capabilities of the Observatory [2], especially for mass composition measurements, and to serve as well as a testbed of new technologies for the next generation of surface-based UHECRs experiments. Radio-based detectors, one of the technologies being explored, promise a signal proportional to the electromagnetic component of the shower that suffers minimal attenuation in the atmosphere and instrumentation that is available with moderate costs. Up to recently, radio detection efforts have concentrated on the coherent radio emission in the MHz band of the spectrum, where the CODALEMA [3] and LOPES [4] experiments have studied in detail the characteristics of the signal and have helped elucidate the emission mechanism that dominates at these wavelengths. The current AERA set-up [2] at the Pierre Auger Observatory, is designed to determine the feasibility of using the $\mathrm{MHz}$ technique to study cosmic rays at the highest energies.

Recent results of a test beam experiment [5] have triggered a renewed interest in the use of the $\mathrm{GHz}$ band of the spectrum for the detection of UHECRs. These measurements with an electron beam at SLAC show a microwave signal emitted in coincidence with the passage of the beam inside an anechoic chamber. This signal is interpreted as molecular bremsstrahlung (MBR) emitted by the interaction of the low energy electrons in the plasma produced by the shower ionization with the molecules of the atmosphere. MBR is expected to be unpolarized and emitted isotropically. The measurements at SLAC show a time constant for the emission process of a few nanoseconds, and a signal level that scales quadratically with the particle content of the beam [5]. These characteristics make MBR in the GHz band a very promising channel for the study of UHECRs, especially since the isotropic emission allows for the

\footnotetext{
ae-mail: facal@kicp.uchicago.edu

${ }^{b}$ For the full authorlist see Appendix "Collaborations" in this volume

This is an Open Access article distributed under the terms of the Creative Commons Attribution License 2.0, which permits unrestricted use, distribution, and reproduction in any medium, provided the original work is properly cited.
} 
observation of the longitudinal shower profile in the same way as done in a Fluorescence Detector (FD). Moreover, atmospheric attenuation in this band is virtually negligible in most cases for the distances involved and the routine use of different sub-bands of the $\mathrm{GHz}$ spectrum for satellite TV emission, wireless telecommunications and others make commercial instrumentation widely available at limited costs.

\section{MICROWAVE DETECTION AT THE PIERRE AUGER OBSERVATORY}

The Pierre Auger Observatory is running a dedicated program aimed at establishing the feasibility of the microwave detection technique [2]. Three different experiments are (or will be) installed at the Observatory site. Their aim is the detection of the microwave signal coming from an extensive air shower in coincidence with the events recorded by the Observatory.

Two of the experiments, AMBER and EASIER are already installed and operating in coincidence with the Auger Surface Detector (SD). MIDAS was commissioned and run in Chicago for a few months and will be re-commissioned in Malargüe in the near future. AMBER and MIDAS share the concept of being radio-fluorescence detectors, telescopes aimed at the distant observation of the shower longitudinal development like an FD. While AMBER is designed to be triggered by the SD, MIDAS has a trigger system that closely follows the design of the trigger of the Auger FD [6]. EASIER, on the other hand, has been projected as a complement to the SD, instrumenting the tanks in the array with an additional radio-detector that is integrated with the tank electronics and data acquisition.

All the experiments work in the extended C-band, between 3.4 and $4.2 \mathrm{GHz}$, that is reserved to the reception of direct broadcast satellite television. The sensitive elements of AMBER are antenna horns that are coupled to a low noise block amplifier and a down-converter unit (LNB). EASIER and MIDAS use LNBFs that integrate the active antenna element and the amplifier into a single compact unit, produced for the reception of satellite TV. In both cases, the amplifiers have a gain $\sim 65 \mathrm{~dB}$ and the down-converted signal a frequency of $\sim 1 \mathrm{GHz}$. This RF signal is then feed to a logarithmic power detector that outputs a DC level proportional to the input power in $\mathrm{dBm}$ with a time response of the order of 10 or $100 \mathrm{~ns}$ (depending on the configuration). The output of the power detector is suitable for digitization in a Flash-ADC (FADC).

\subsection{AMBER}

AMBER is a radio telescope instrumented with a $2.5 \mathrm{~m}$ off-axis parabolic dish that images an approximately $7^{\circ} \times 7^{\circ}$ section of the sky onto a camera segmented in 16 pixels (Fig. 1). The four central pixels are double polarized dual C-band and Ku-band (10.9-14.5 GHz) feed-horns. The 12 outer pixels are single polarized C-band feed-horns. Amplification and down-conversion of the signal takes place in a low noise block (LNB) attached to each one of the feed-horns. The downconverted signal is then fed into a logarithmic power detector that is integrated in a compact-PCI card that contains also the digital electronics, including the FADC that digitizes the signal with $100 \mathrm{MHz}$ sampling rate.

The digital electronics is designed to accommodate the $\sim 3 \mathrm{~s}$ latency of the external trigger received from the surface detector. The FADC samples are held in a large circular memory buffer capable of storing up to $5 \mathrm{~s}$ of data for each channel. Upon reception of an SD trigger, the approximate time of the shower in the field of view of AMBER is calculated from the preliminary geometrical reconstruction of the SD event and $100 \mu$ s of data are selected from the buffer and stored for further analysis. The length of the readout window takes into account the uncertainty of $500 \mathrm{~m}$ and $10^{\circ}$ in the online reconstruction of the core position and direction of the shower.

The AMBER feeds were calibrated using a liquid nitrogen bath. The contribution of the dish to the noise temperature was then measured using a calibrated LNB. The combined noise temperature of the system in the $\mathrm{C}$-band ranges from $45 \mathrm{~K}$ for the outer pixels to $65 \mathrm{~K}$ for the inner ones. AMBER is installed alongside the High Elevation Fluorescence Telescopes (HEAT) overlooking the infill SD area 

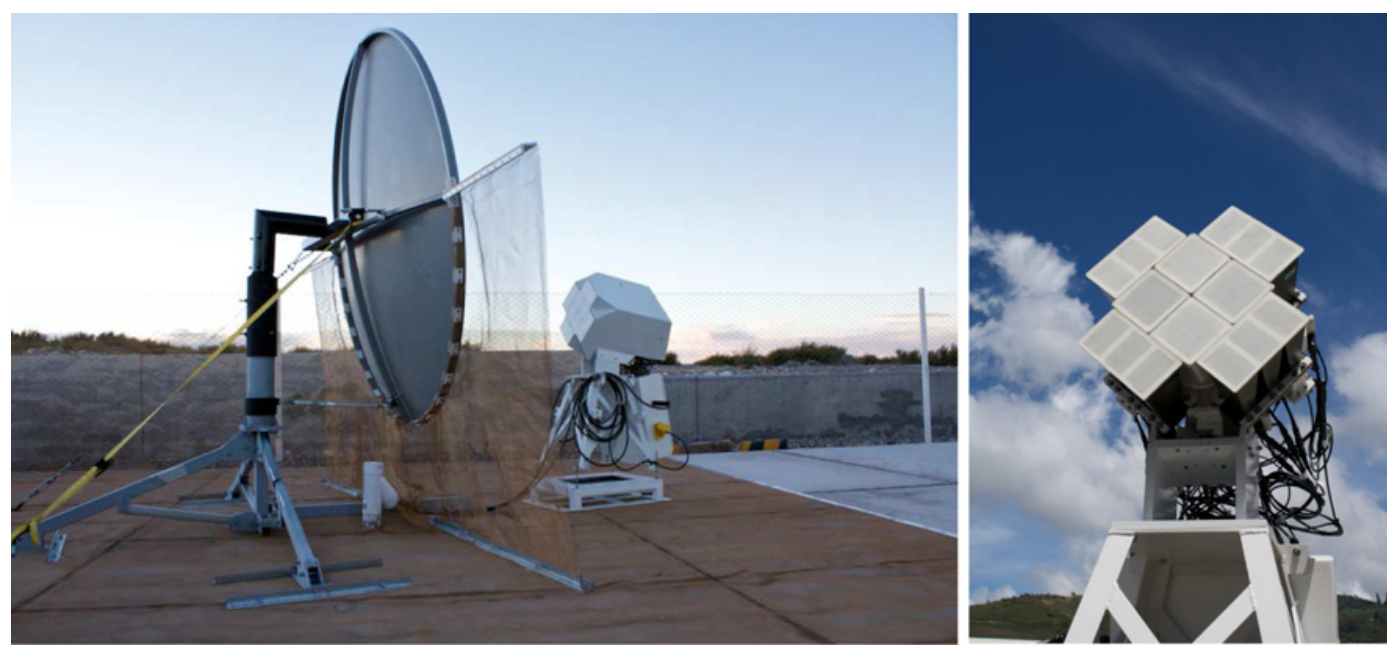

Figure 1. The AMBER telescope at the HEAT site. The $2.5 \mathrm{~m}$ off-axis parabolic dish (left) focuses the incoming signal onto a 16 pixel camera (right).

(the region were the SD detectors are closer together to study lower energy showers). Commissioning took place in 2011. During commissioning several transits of the Sun and the Crab nebulae were used to validate the absolute calibration and the optical performance of the detector. Data taking and analysis are now underway.

\subsection{EASIER}

EASIER is an alternative design to the radio-telescope adopted by AMBER and MIDAS. Each SD tank is instrumented with a wide field of view $\left(\sim 60^{\circ}\right)$ radio receiver. The receivers used by EASIER have small effective area $\left(3 \cdot 10^{-3} \mathrm{~m}^{2}\right)$ that is compensated in this case by closer distances to the shower (of the order of one $\mathrm{km}$, as opposed to 5 or $10 \mathrm{~km}$ in the case of a telescope) and by the compression of arrival time of the signal when the shower is observed from closer to its axis (that can result in a factor 10 of increase in the signal).

The tanks are instrumented with a commercial LNBF mounted on top of a $3 \mathrm{~m}$ mast and aligned in the direction of the zenith (Fig. 2). The output of the LNBF is measured with a power detector as a DC pulse of amplitude proportional to the RF power. This pulse, after being properly adapted to the input characteristics of the tank FADC, is digitized using one of the available channels of the tank electronics board. In this way, the EASIER signal represents an additional data source for the tank: the antenna is triggered when the tank is triggered, and its output data is integrated into the SD data stream.

In a first phase the EASIER project consisted of seven instrumented tanks (a central tank plus the surrounding hexagon), deployed in April 2011. This first instrumented hexagon yielded the first evidence of $\mathrm{GHz}$ emission by air showers (Sec. 3). In order to improve significantly the available statistics, 54 additional tanks were instrumented in April 2012, bringing the total EASIER tanks to 61. We expect of the order of 1 event/month in the upgraded EASIER configuration (with the signal in [5] scaled linearly with the shower energy).

\subsection{MIDAS}

MIDAS was designed as a prototype of a radio- $F D$, a radio telescope capable of observing the shower longitudinal profile, and capable of triggering on the $\mathrm{GHz}$ signal from an extensive air shower. In its first 


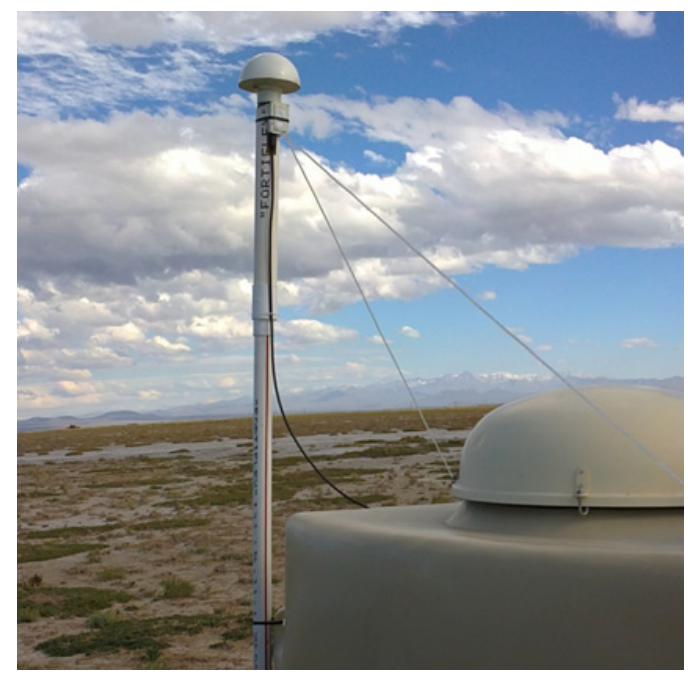

Figure 2. EASIER antenna installed in a tank. The LNBF unit completed with a radome weather cover is installed on top of a $3 \mathrm{~m}$ mast and is oriented towards the zenith.
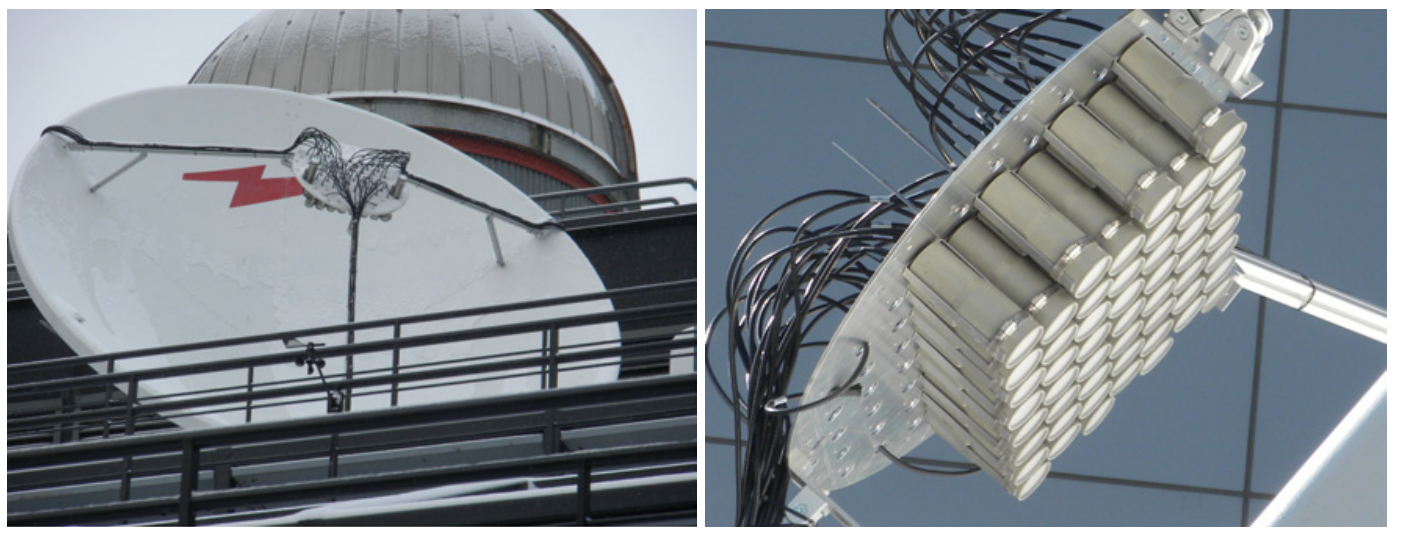

Figure 3. The MIDAS telescope assembled at the roof of the Physics teaching building of the University of Chicago (left). The telescope camera is instrumented with 53 LNBFs arranged to maximize coverage over the field of view (right).

configuration MIDAS was installed at the University of Chicago campus, using a $4.5 \mathrm{~m}$ parabolic dish as a reflector and equipped with a 53 channel camera [7]. It is planned that MIDAS will be recommissioned at the Auger Observatory using a $5 \mathrm{~m}$ parabolic dish installed near the FD station at Los Leones.

Each one of the 53 channels of MIDAS is instrumented with a single polarization C-band LNBF. The RF signal at the output of the LNBF is measured in a power detector that is then sampled using a custom built 14-bit $20 \mathrm{MHz}$ FADC module [8]. This VME module is equipped with an FPGA that implements the first level trigger (FLT). This pixel trigger is based on the running sum of 20 FADC samples and each pixel has as self regulated trigger threshold that keeps its trigger rate at $100 \mathrm{~Hz}$. The second level trigger (SLT) algorithm searches then for FLT pixels compatible in time and space with the expected trace of a cosmic ray shower, using a set of pre-defined camera patterns. When an SLT is found, 2000 FADC samples per channel (including 500 pre-trigger samples) are readout and stored 


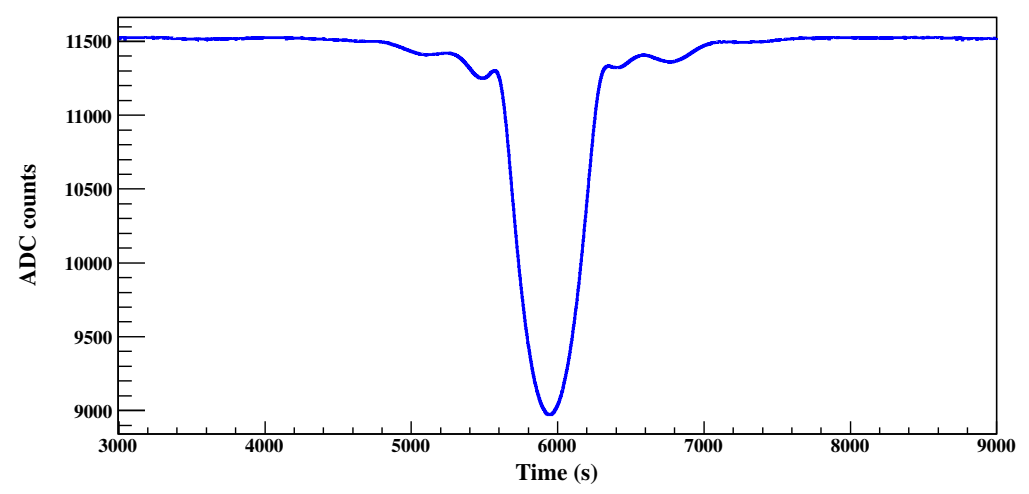

Figure 4. A sun transit in the field of view in the MIDAS central pixel. The minimum corresponds to the moment when the Sun is fully contained in the field of view of the pixel and allows to establish the absolute calibration of the telescope.

for offline analysis. A patch panel antenna placed at the center of the parabola is fired each 15 minutes during data taking to monitor the correct operation and stability of the trigger system.

The Sun, whose absolute flux at $\mathrm{GHz}$ is monitored daily from different radio-observatories, is used as the main calibration source (Fig. 4). From repeated runs of the sun calibration and accounting for the uncertainty on the absolute pointing of the telescope, we obtain a mean value of the system temperature of $T_{s y s}=65 \pm 3 \mathrm{~K}$, for an effective area $A_{e f f}=9.1 \mathrm{~m}^{2}$ (calculated with a detailed electromagnetic simulation of the set-up).

The MIDAS prototype has been run in data taking mode for about three months. Some event candidates were found during the run, but their signals were too close to the background for properly estimating their significance given the harsh RF conditions of the Chicago site. The absence of candidates of 5 pixels or more, after a dedicated event search and a detailed Monte Carlo simulation of the expected signals has allowed us to place limits on the GHz emission from UHECRs showers [9]. Once installed at the Auger site, the expected event rate is $\sim 1$ event/month with maximum sensitivity at $\sim 10^{19} \mathrm{eV}$ (with the flux in [5] scaled linearly with the shower energy).

\section{RESULTS: FIRST EVIDENCE OF GHZ EMISSION FROM AN EXTENSIVE AIR SHOWER}

The EASIER experiment has produced the first unambiguous evidence of the emission of GHz radiation by an air shower, with the 6-tank prototype instrumented in 2011 (Fig. 5). A signal of $14 \sigma$ significance has been measured (Fig. 6, left panel) in coincidence with an air shower landing in the array. The shower had an energy of $14 \mathrm{EeV}$, as measured by the $\mathrm{SD}$ and a zenith angle of $30^{\circ}$. The shower landed at a distance of $140 \mathrm{~m}$ of the nearest station, the one that recorded the $\mathrm{GHz}$ signal and that was the one at the center of the hexagon instrumented with radio receivers. The signal in the $\mathrm{GHz}$ antenna arrived slightly before the signal of the photomultiplier tube (PMT) in the tank, which excludes the possibility of a spurious trace induced by the PMT itself. The other six antennas of the instrumented hexagon did not register a signal (Fig. 6, right panel).

The resulting signal is compatible with what we expect using the signal measured in [5] and scaling it linearly by the relation between the energy of the measured shower and the shower equivalent energy for the test beam measurements $\left(3.17 \cdot 10^{17} \mathrm{eV}\right)$, after accounting properly for the shower geometry and the characteristics of the EASIER set-up. A similar calculation for the expected Cherenkov signal (without including coherence effects over the Cherenkov emission by the electrons in the shower) cannot account for the measured signal. This however does not mean that the signal can be attributed to MBR since 


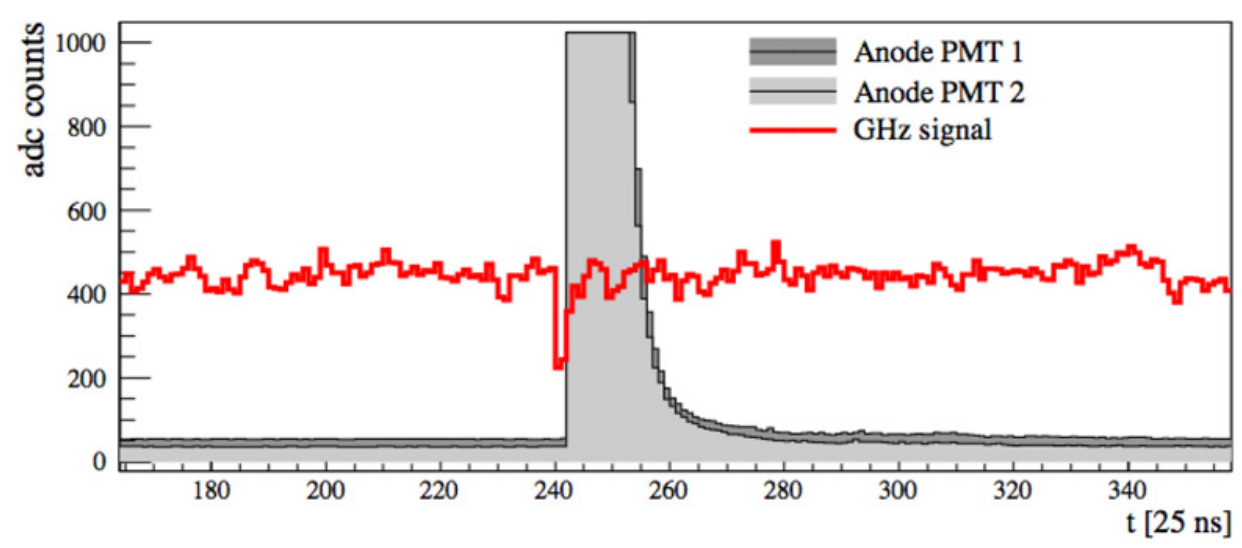

Figure 5. Signal recorded by the EASIER antenna (red) in coincidence with the anode signal in two of the PMTs of same tank (gray, shadowed) for a $14 \mathrm{EeV}$ event in June 2011.
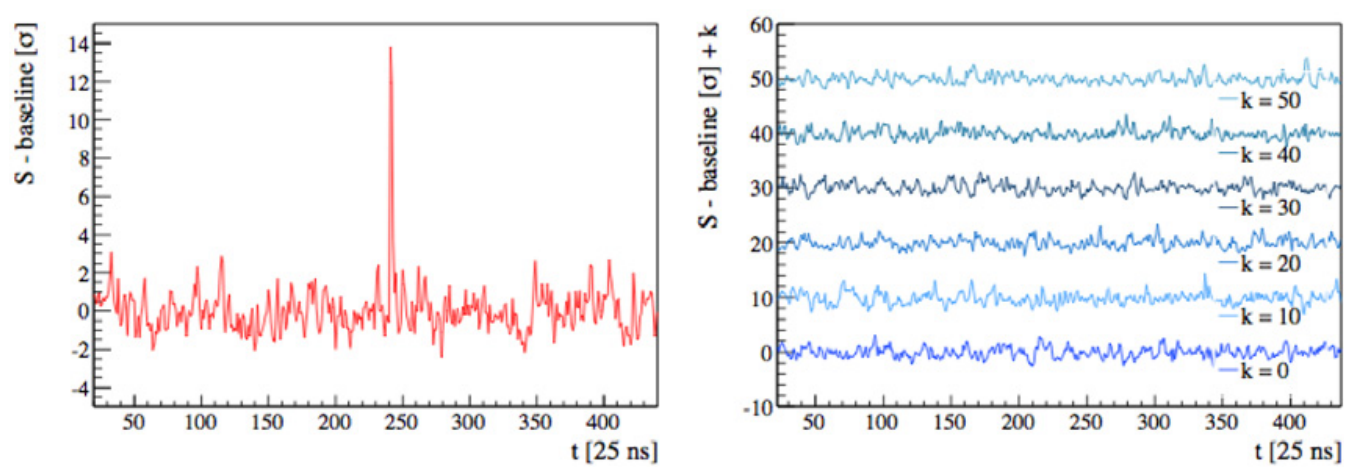

Figure 6. Left panel: the signal measured in the EASIER antenna, expressed in terms of the $\sigma$ of the baseline fluctuations. The maximum has a significance of $14 \sigma$. Right panel: same for the rest of the antennas in the instrumented hexagon (for clarity the different traces are displaced by a constant term, $k$, as indicated).

we cannot discard other processes that could contribute to the signal especially since some portions of the shower have been observed close to the Cherenkov angle that sets scale for any coherent effect that could enhance these contributions.

\section{CONCLUSIONS}

The Pierre Auger Observatory is running a strong program for the microwave detection of UHECRs. Two experiments, AMBER and EASIER are currently installed at the Pierre Auger Observatory with a third, MIDAS, to follow. The design of a fourth, FDWave [2] is being finalized. Evidence of the emission of $\mathrm{GHz}$ radiation in an air shower has already been found with the EASIER experiment. This result has been confirmed by the CROME experiment [10].

The objective of the program is now to establish the nature and the characteristics of the $\mathrm{GHz}$ emission. We expect that the information gathered at the different set-ups within the Observatory, together with the one coming from the new generation of test beam experiments (like AMY [11] and MAYBE [12]) will allow us to establish if GHz radiation will be an useful tool for the study of UHECRs. 


\section{References}

[1] The Pierre Auger Collaboration, Nucl. Instrum. Meth. A 523 (2004) 50

[2] The Pierre Auger Collaboration, in Proc. 32nd ICRC, Beijing, China (2011), arXiv:1107.4807 [astro-ph]

[3] D. Ardouin et al., Astropart. Phys. 31 (2009) 192, arXiv:0901.4502 [astro-ph]

[4] The LOPES Collaboration, Nature 435 (2005) 313, astro-ph/0505383

[5] P.W. Gorham et al., Phys. Rev. D 78 (2008) 032007, arXiv:0705.2589 [astro-ph]

[6] The Pierre Auger Collaboration, Nucl. Inst. Meth. A 620 (2010) 227, arXiv:0907.4282 [astro-ph]

[7] P. Facal San Luis et al., Nucl. Instrum. Meth. A 662 (2012) S118

[8] M. Bogdan et al., in Proc. of the 2011 IEEE Nuclear Science Symposium Conference (2011) 1252

[9] J. Alvarez-Muniz et al., submitted to Phys. Rev. Lett., arXiv:1205.5785 [astro-ph]

[10] R. Smida et al., these proceedings

[11] V. Verzi et al., these proceedings

[12] C. Williams et al., these proceedings 